\title{
Gradenigo Syndrome as the Form of Presentation of Nasopharyngeal
}

\author{
Elham Ouspid, ${ }^{1}$ and Payam Sariaslani ${ }^{1, *}$ \\ ${ }^{1}$ Department of Neurology, Emam Reza Hospital, Kermanshah University of Medical Sciences, Kermanshah, IR Iran \\ "Corresponding author: Payam Sariaslani, Department of Neurology, Emam Reza Hospital, Kermanshah University of Medical Sciences, Kermanshah, IR Iran. E-mail: \\ samira.gavam@yahoo.com
}

Received 2015 April 30; Accepted 2016 February 16.

\begin{abstract}
Nasopharyngeal carcinoma is rare in many parts of the world, but is prevalent among Southeast Asians. Usual presenting symptoms include recurrent epistaxis, otitis media and headache. Nasopharyngeal carcinoma may also mimic Gradenigo syndrome, which consists of otitis media, facial pain in the regions innervated by the first and second divisions of trigeminal nerve and abducens nerve paralysis. In this report, we present a case which was initially diagnosed as gradenigo syndrome but turned out to be an invasive nasopharyngeal carcinoma.
\end{abstract}

Keywords: Nasopharyngeal Carcinoma, Gradenigo Syndrome, Abducens Palsy

\section{Introduction}

Gradenigo syndrome consists of otitis media, facial pain in the region of first and second division of trigeminal nerve and abducens nerve paralysis. Cranial nerve dysfunction is caused by otitis and local leptomeningitis near the apex of the petrous bone, where the trigeminal nerve ganglion and the abducens nerve lay closely together [1]. Nasopharyngeal carcinoma (NPC) usually presents with intermittent epistaxis, hearing impairment, headache, adult-type serous otitis media, and pain in the head and neck. Single or multiple cranial nerve palsies also may be the initial presentation [2]. NPC may also mimic Gradenigo syndrome, since it can obstruct the eustachian tube, resulting in serous otitis media and invade the cavernous sinus, causing sixth nerve paresis [3]. We present a case which was initially diagnosed as gradenigo syndrome but turned out to be an invasive NPC.

\section{Case Presentation}

A 65-year-old hypertensive woman was referred with lo day history of diplopia, facial pain and hearing disturbance. She had a history of postnasal discharge, headache and otalgia approximately 45 days before admission. She developed right side stabbing neuralgiform headache seven days later and diplopia 10 days prior to admission. On admission there was mild tenderness on right mastoid bone, hypesthesia on territory of ophthalmic and maxillary divisions of right fifth nerve, right abducens nerve palsy and conductive hearing loss on right side. The rest of the exam was otherwise normal. On account of the prior history of upper respiratory tract infection, hearing loss and right side mastoid tenderness in association with right sixth and fifth nerve palsy a diagnosis of Gradenigo syndrome was considered and intravenous antibiotic therapy was started. The initial axial CT scan was not informative and was reported normal. Contrast enhanced brain MRI (Figure 1) revealed right side mastoiditis and otitis, and an enhancing mass lesion $36 \times 27 \mathrm{~mm}$ in diameter in roof of nasopharynx, with destruction and invasion to sphenoid sinus, clivus, right petrous bone and right cavernous sinus. CT scanning of bilateral temporal bone and base of skull (Figure 2) confirmed a diagnosis of nasopharyngeal mass. The patient underwent endoscopic biopsy and pathologic examination of the mass revealed a squamous cell NPC. Chemotherapy regiment was started, but about 4 months later patient expired due to fulminant sepsis.

\section{Discussion}

NPC may present with various symptoms including nasal obstruction, rhinorrhea, epistaxis and serous otitis media [4]. It is unique among head and neck cancers in its propensity to involve cranial nerves, partly owing to the proximity of the nasopharynx to the base of skull [5]. It may also mimic Gradenigo syndrome, since it can obstruct the eustachian tube, resulting in serous otitis media, and invade the cavernous sinus and cause fifth and sixth nerve paresis [3]. In 2001 a 53 year old patient was reported from Spain presenting with gradenigo syndrome (involvement 


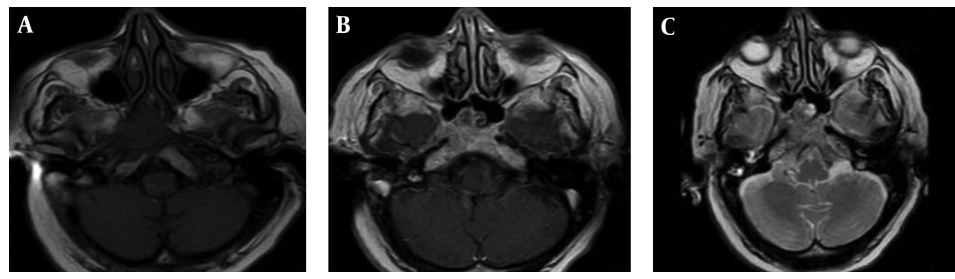

Figure 1. Axial Magnetic Resonance Images, Showing the Nasopharyngeal Mass With Skull Base Destruction on A, T1 Weighted Image and B, Post Contrast Enhancement on Contrast Enhanced T1 Weighted Image; C, the Erosive Mass is Also Visualized on T2 Axial View
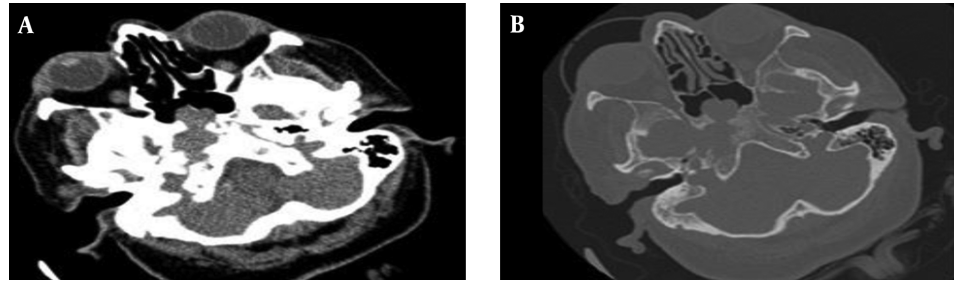

Figure 2. Temporal Bone CT Scans With A, Tissue and B, Bone Windows Showing the Origin and Course of Invasion of the Nasopharyngeal Carcinoma

of right fifth and sixth cranial nerve), who's otorhinolaryngological assessment led to diagnosis of a squamous cell NPC. On imaging there was extension to the skull base and adjacent right cavernous sinus [6].

In 2006 Kumar Jana et al. presented an almost similar case of a 15 year old Indian boy presenting with gradenigo syndrome that was diagnosed initially to be due to left side cholesteatoma, but several days after mastoid exploration the patient developed involvement of other cranial nerves and the histopathlogic examination revealed undifferentiated NPC [7]. Epstein and Jones reviewed 52 patients with a histologic diagnosis of a carcinoma of the nasopharynx; in 20 of the patient's the initial diagnosis was incorrect. A diagnosis of a middle ear infection was made in 10 patients, other type of ear, nose, or throat infection in 7 patients, sinusitis in 1 patient, allergy in 1 patient and TM joint disease in 1 patient. There was a mean delay of 8.3 months from the initial signs and symptoms to the final diagnosis of NPC [8]. Diagnosis of NPC requires a high index of suspicion in view of its initial sparse, non-specific symptoms. Ideally the disease should be detected in its early stages, regarding high potential of focal invasion and distant metastasis, and great potential for cure if diagnosed early in its course [8]. We believe that it is useful to recommend that in cases of Gradenigo syndrome a full systematic otorhinolaryngological exploration be made to effectively rule out this disorder.

We recommend that baseline evaluation of cranial nerve palsies be extended to include consultation with an otolaryngologist if there are sign or symptoms of nasopha- ryngeal or ear involvement.

\section{Acknowledgments}

We appreciated Gaem medical laboratory of Ilam city (Iran) for diagnosis helps.

\section{Footnotes}

Conflict of Interests: The authors declare no conflict of interest.

Funding/Support: Kermanshah University of Medical Sciences.

\section{References}

1. Villa G, Lattere M, Rossi A, Di Pietro P. Acute onset of abducens nerve palsy in a child with prior history of otitis media: a misleading sign of Gradenigo syndrome. Brain Dev. 2005;27(2):155-9. doi: 10.1016/j.braindev.2004.02.003. [PubMed: 15668058].

2. Chen JF, Lee ST. Nasopharyngeal carcinoma presenting as an intracranial abscess. Surg Neurol. 1998;49(5):553-7. [PubMed: 9586935].

3. Azarmina M, Azarmina H. The six syndromes of the sixth cranial nerve. J Ophthalmic Vis Res. 2013;8(2):160-71. [PubMed: 23943691].

4. Dare AO, Gibbons KJ, Proulx GM, Fenstermaker RA. Resection followed by radiosurgery for advanced juvenile nasopharyngeal angiofibroma: report of two cases. Neurosurgery. 2003;52(5):1207-11. [PubMed: 12699567] discussion 1211.

5. Leung SF, Tsao SY, Teo P, Foo W. Cranial nerve involvement by nasopharyngeal carcinoma: response to treatment and clinical significance. Clin Oncol (R Coll Radiol). 1990;2(3):138-41. [PubMed: 2261400]. 
6. Penas-Prado M, Diaz-Guzman J, Jimenez-Huerta I, Juntas-Morales R, Villarejo-Galende A, Diez-Torres I. [Gradenigo syndrome as the form of presentation of nasopharyngeal carcinoma]. Rev Neurol. 2001;32(7):638-40. [PubMed: 11391492].

7. Jana AK, Jaswal A, Sikder B, Jana U, Nandi TK. Nasopharyngeal car cinoma presenting as Gradenigo's syndrome. Indian J Otolaryngol
Head Neck Surg. 2006;58(4):400-2. doi: 10.1007/BF03049612. [PubMed: 23120365].

8. Epstein JB, Jones CK. Presenting signs and symptoms of nasopharyngeal carcinoma. Oral Surg Oral Med Oral Pathol. 1993;75(1):32-6. [PubMed: 8419872]. 